\title{
Adverse childhood experiences and trajectories of internalizing, externalizing, and prosocial behaviors from childhood to adolescence
}

\author{
Leonardo Bevilacqua $^{\mathrm{a}, *}$, Yvonne Kelly ${ }^{\mathrm{a}}$, Anja Heilmann ${ }^{\mathrm{a}}$, Naomi Priest ${ }^{\mathrm{b}, \mathrm{c}}$, \\ Rebecca E. Lacey ${ }^{\mathrm{a}}$ \\ ${ }^{a}$ Research Department of Epidemiology and Public Health, University College London, 1-19 Torrington Place, WC1E 6BT, London, United Kingdom \\ ${ }^{\mathrm{b}}$ Australian National University, Centre for Social Research and Methods, Australian National University, Canberra, Australia \\ ${ }^{\mathrm{c}}$ Population Health, Murdoch Children's Research Institute, The Royal Children's Hospital, Melbourne, Australia
}

\section{A R T I C L E I N F O}

\section{Keywords:}

Adverse childhood experiences

Cohort study

Externalizing

Internalizing

Millennium Cohort Study

Prosocial

Behavior

ACE score

Individual ACEs

\begin{abstract}
A B S T R A C T
Background: Adverse childhood experiences (ACEs) predict poorer mental health across the life course but most of the extant research has employed ACE scores or individual adversities using retrospective data.

Objectives: To study the impact of ACEs on later mental health using not only ACEs scores and individual ACEs, but also latent class analysis (LCA), which respects the clustering of adversities. Participants and setting: 8823 members from the UK Millennium Cohort Study.

Methods: We investigated the impact of prospectively reported ACEs on mental health trajectories derived using the Strengths and Difficulties Questionnaire at age 3, 5, 7, 11 and 14. Associations between LCA-derived ACE clusters, ACE scores, individual ACEs and mental health trajectories were tested using linear mixed effects models.

Results: With statistical significance set at 5\% level, ACE scores showed a graded association with internalizing (ACE score of $1: \beta=0.057$; ACE score of 2 : $\beta=0.108$; ACE score of 3 : $\beta=0.202$ ), externalizing (ACE score of $1: \beta=0.142$; ACE score of $2: \beta=0.299$; ACE score of 3 : $\beta=0.415$ ) and prosocial behaviors (ACE score of 1: $\beta=-0.019$; ACE score of 2 : $\beta=-0.042$; ACE score of 3: $\beta=-0.059)$. Harsh parenting and physical punishment were particularly strongly associated with externalizing $(\beta=0.270$ and $\beta=0.256)$ and negatively associated with prosocial behaviors ( $\beta=-0.046$ and $\beta=-0.058)$. Parental discord and parental depression showed the strongest associations with internalizing problems $(\beta=0.125$ and $\beta=0.113)$. LCA did not discriminate ACE clusters in this dataset.

Conclusions: ACEs have an important impact on mental health from childhood to adolescence. ACEs score approach yielded useful results, which were further enhanced by exploring individual ACEs.
\end{abstract}

Abbreviations: ACEs, Adverse Childhood Experiences; LCA, Latent Class Analysis; MCS, Millennium Cohort Study.

* Corresponding author.

E-mail address: l.bevilacqua@ucl.ac.uk (L. Bevilacqua). 


\section{Introduction}

Adverse childhood experiences (ACEs) can be broadly defined as "those experiences which require significant adaptation by the developing child in terms of psychological, social and neurodevelopmental systems, and which are outside of the normal expected environment" (McLaughlin, 2016). These include, amongst others, child maltreatment and abuse, parental conflict and separation, and parental psychiatric disorders. The importance of ACEs for poor lifelong health and mental health outcomes has been well documented (Hughes et al., 2017) and given that the impact of ACEs is both significant and long-term, ACEs are extremely costly for both individuals and society as a whole. For instance, ACEs were recently forecasted to cost Europe and North America \$581 billion/year and $\$ 748$ billion/year, respectively (Bellis, Lowey, Leckenby, Hughes, \& Harrison, 2014).

\subsection{ACEs and internalizing, externalizing, and prosocial behaviors}

Earlier research into ACEs indicated a dose-response relationship between the number of ACEs (an 'ACE score') and several health and mental health conditions, such as obesity, alcohol and drug abuse, depression and suicide (Anda et al., 2006; Felitti et al., 1998). More recent studies confirm a consistently strong, and positive association between retrospective reports of adversities in childhood and several adult mental health problems such as internalizing problems (anxiety and depression), psychosis, substance misuse, and personality disorders (Carr, Martins, Stingel, Lemgruber, \& Juruena, 2013; Hengartner, Ajdacic-Gross, Rodgers, Muller, \& Rossler, 2013; Kalmakis \& Chandler, 2015). In addition, ACEs have been linked to violence in dating relationships (E. Miller et al., 2011) and violence and incarceration in adults (Bellis et al., 2014); In this last study, the authors highlighted that the relationship between ACEs and antisocial behavior was particularly marked, and explained that $52 \%$ of adult violence perpetration might be attributed to ACEs.

A growing body of evidence has highlighted that the negative impact of ACEs can be seen earlier, in children and adolescents (Scully, McLaughlin, \& Fitzgerald, 2020). Similar to adult studies, the occurrence of ACEs has been related to child and adolescent externalizing such as hyperactivity and conduct problems (Appleyard, Egeland, van Dulmen, \& Alan Sroufe, 2005; Baglivio et al., 2014) and internalizing behaviors (Flouri \& Panourgia, 2011), including manifestations such as adolescent suicide (Borges, Angst, Nock, Ruscio, \& Kessler, 2008; Goodday, Shuldiner, Bondy, \& Rhodes, 2019). Though most of the research in this field has looked at males and females together, there is evidence that ACEs affect boys and girls differently e.g. females are more likely to experience sexual abuse while boys are more likely to experience verbal abuse (Cunningham et al., 2014), but also that the same ACEs may have a different impact across males and females e.g. sexual and verbal abuse during childhood were significantly associated with smoking for women but not for men (Fuller-Thomson, Filippelli, \& Lue-Crisostomo, 2013).

While there are several studies on the impact of ACEs on internalizing and externalizing behaviors in both young people and adults, an area that has been less studied in relation to ACEs is their potential impact on adaptive behaviors such as the development of prosocial behaviors (e.g. helping others, sharing, and being kind to peers). Previous studies concerning children's social competence and prosociality have often been cross-sectional, thereby providing little information about their developmental significance. Despite the argument that social competence may be a multi-dimensional construct (Schneider, Ackerman, \& Kanfer, 1996), researchers have focused mainly on either the broad construct of positive or adaptive functioning, or on one of the relevant dimensions of social competence (Masten et al., 1995; Robins, John, Caspi, Moffitt, \& Stouthamer-Loeber, 1996). Available longitudinal studies, however, highlight that prosocial behavior is predictive of educational achievement, well-being and adjustment (Nantel-Vivier, Pihl, Côté, \& Tremblay, 2014). Despite the relevance of ACEs in predicting several life outcomes, few studies have investigated whether ACEs may delay or hinder the development of prosocial behavior, which is itself predictive of psychosocial adjustment (Eisenberg, Fabes, \& Spinrad, 2006).

\subsection{Approaches to the study of ACEs and limitations of extant research}

Although the ACEs score approach has been widely applied in ACEs and physical health and mental health research over the past 40 years (Chapman et al., 2004; Chen \& Lacey, 2018; Rasmussen et al., 2019; Slopen et al., 2015), there are several issues with this approach when investigating associations with health and mental health (Rebecca E Lacey \& Minnis, 2020). The most notable of these is the assumption that each adversity is equally important for the outcome of interest and the specific patterning of co-occurring ACEs is ignored. For instance, the combination of parental mental illness and parental separation (ACE score of 2) is treated as the same as physical and sexual abuse (also an ACE score of 2). An alternative to the ACEs score approach is to explore the effects of individual adversities. There are several studies which examined the association of single adversities on mental health and behavior (Hillberg, Hamilton-Giachritsis, \& Dixon, 2011; Luecken \& Roubinov, 2012). The findings suggest that individual adversities show different associations with mental health and behavior. For example, children and young people who experience parental divorce are more likely to display externalizing disorders, substance abuse, and low educational attainment (Hailey Maier \& Lachman, 2000), whereas internalizing disorders and lower self-confidence are more prominent among bereaved youth (Mack, 2001). Whilst looking at single adversities might be informative in teasing out the mechanisms involved (Rebecca E. Lacey, Bartley, Pikhart, Stafford, \& Cable, 2014), the effects of that adversity might be confounded by the experience of other adversities which have not been accounted for in the analyses.

Given the increasing recognition of the limitations of ACE scores and single adversity approaches in ACEs research, there has been an emergence of alternative methods of operationalising ACEs which respect their clustering and are potentially more informative when looking at associations with health and behavior. These alternative analytic approaches have included person-centred methods, such as Latent Class Analysis (LCA). LCA is a data-driven approach which aims to identify distinct groups or classes of individuals who 
have similar patterns of reported adversities (Masyn, 2013). The use of LCA with ACEs data has increased in the past few years and allows the researcher to explore whether the specific patterning of ACEs is important for future outcomes. Several studies have explored whether ACE classes obtained using LCA show differing associations with health and mental health outcomes. In a sample of German children aged 4-17 years, Witt et al. (2016) identified three maltreatment classes using LCA - "Multiple types of maltreatment excluding sexual abuse" (63.1\%), "Multiple types of maltreatment including sexual abuse" (26.5 \%) and "Predominately sexual abuse $(10.3 \%)$ ". The second class showed the poorest health outcomes in terms of mental disorders and health-related quality of life suggesting that the combination of sexual abuse with other maltreatments (physical and psychological abuse, witnessing domestic violence and neglect) was particularly detrimental to health. Ho, Chan, Chien, Bressington, and Karatzias (2019) also identified three ACE classes among university students in Hong Kong - "Low ACEs" (76.0 \%), "Household violence" (20.6 \%) and "Household dysfunction" (3.4\%). Students in the "Household violence" class - characterised by high probability of reporting physical and psychological abuse and domestic violence - were more likely to report depression and maladjustment symptoms than those in the "Low ACEs' class.

\subsection{The present study}

In the present study we will employ all three approaches described above (ACE scores, individual ACEs, LCA derived ACE clusters) to study associations with mental health in childhood and into adolescence, focussing on internalizing and externalizing behaviors. Also, we will use these three different strategies to study how ACEs are associated with prosocial behavior in childhood and into adolescence - an area that has been overlooked despite its importance in predicting life adjustment and positive outcomes. In addition, we will investigate the impact of ACEs on internalizing, externalizing, and prosocial behavior using 5 different time points, from childhood to adolescence. This will allow us to have a more thorough understanding of whether ACEs impact internalizing, externalizing, and prosocial behavior across childhood and adolescence, instead of using a single time-point. We also derived our ACE measures from prospectively reported data. Many previous studies have utilised retrospectively reported ACEs, which are known to have important limitations (Danese, 2020). Finally, we explored whether associations between ACE scores, individual ACEs, and LCA-derived ACE clusters with early life mental health differed for boys and girls. We further extend the literature by applying these approaches using a UK-wide nationally-representative cohort study - the Millennium Cohort Study.

We hypothesise that ACE scores will show a dose-response effect on the three outcomes considered (i.e. with a higher number of ACEs having a more negative impact on our outcomes). In terms of individual ACEs or clusters of ACEs, we hypothesise that harsh parenting and forms of physical abuse (i.e. physical punishment) would be strongly associated with worse outcomes across early life. We also hypothesise that ACEs will cluster in the Millennium Cohort Study and LCA will derive distinct groups of children who experienced similar ACEs. Finally, we anticipate that associations will differ between girls and boys in that associations between ACEs and internalizing behaviors will be stronger for girls and associations with externalizing behaviors stronger for boys.

\section{Methods}

\subsection{Participants}

The MCS is a longitudinal survey following a nationally representative, clustered and stratified sample of 19,000 children born in the UK in 2000-2001 (Connelly \& Platt, 2014). The sample was drawn from all babies born between 1 September 2000 and 31 August 2001 in England and Wales; those born in Scotland and Northern Ireland between 23 November 2000 and 11 January 2002 . It was selected from a random sample of electoral wards, disproportionately stratified to ensure adequate representation of all four UK countries, of deprived areas and areas with high concentrations of ethnic minority families. The MCS has been tracking the cohort members since the age of nine months and survey data has been collected on six different occasions (i.e. age nine months, three, five, seven, eleven, and fourteen years). Ethical approval for all waves of the MCS was obtained via the NHS Research Ethics Committee system and informed consent obtained from all participants (Shepherd, 2012).

\subsection{Measures}

ACEs: We included information on eight frequently studied ACEs. Parental separation: Data on whether parents were still together were obtained when the cohort member (CM) was 9 months and 3 years. Parents who were reported as divorced or separated at either wave were coded as 'parental separation'. Parental depression/anxiety: data regarding whether a doctor had diagnosed either parent with depression or serious anxiety were collected when the CM was 9 months and 3 years. Data were combined to create a binary variable (if either the mother or father received a diagnosis when the CM was either 9 month or 3 years, then this would be considered a "case"). Parental drug use: a question regarding frequency of drug use was asked to both parents when the CM was 3 years old. Possible answers were "Occasionally", "Regularly", "Never". We combined the responses to make a binary variable ("Occasionally" and "Regularly" = Yes, "Never" = No). Parental alcohol misuse: information regarding the frequency of alcohol use was collected from parents at 9 months and 3 years. The possible answers were: "Every day", " $5-6$ times per week", " $3-4$ times per week", " $1-2$ times per week", "1-2 times per month", "Less than once a month", "Never". Responses were combined to create a binary variable ("Every day" $=$ Yes, all other responses = No). Inter-parental use of force: information whether "Partner ever used force" was collected from both mothers and fathers when CM was 9 months and 3 years. Parents could answer "Yes" or "No". We excluded cases who responded "Don't want to answer". A binary variable was created (if either mother or father answered "Yes" when CM was either 9 month or 3 
years, then this would be considered a "case"). Parental discord: seven questions were asked to both mothers and fathers from the Golombok Rust Inventory of Marital State (GRIMS) scale when the CM was 3 years old (Rust, Bennun, Crowe, \& Golombok, 1986). Although the full scale was not available in MCS, the seven items available ("My husband/wife is usually sensitive to and aware of my needs", "My husband/wife doesn't seem to listen to me", "I sometimes feel lonely even when I am with my husband/wife", "Our relationship is full of joy and excitement", "I wish there was more warmth and affection between us", "I suspect we may be on the brink of separation", "We can always make up quickly after an argument") demonstrated excellent construct validity (Cronbach's alpha $=0.96$ ). Each of the above items was scored 0 or 1 (an affirmative response to the statement). A summary score was obtained by summing the binary scores from all GRIMS items. Those with a summary score of 3 or above were then considered to demonstrate "parental discord". Harsh parenting: six questions from the Straus' conflict tactics scales were asked to mothers when participants were aged 3 (Straus, 1979; Straus, Hamby, Finkelhor, Moore, \& Runyan, 1998). Although the full scale was not available in MCS, there were 6 items available ("How often do you do the following when child is being naughty: ignore him/her", "Shout at him/her", "Send him/her to his/her bedroom or naughty chair", "Take away treats", "tell him/her off", "Bribe him/her"). Responses "Once a month", "Once a week or more", and "Daily" were coded as "Yes", whereas responses "Never" and "Rarely", were coded as "No". The items demonstrated excellent construct validity (Cronbach's alpha $=0.92$ ). A summary score was obtained by summing the binary scores from all Straus' conflict scale items. Those with a summary score of 5 or above were then considered to demonstrate to be suffering "harsh parenting". Physical punishment: Mothers were asked if they "Smacked child when being naughty" when the CM was 3 years old. The response categories were "Never", "Rarely", "Once a month", "Once a week or more", "Daily". We combined responses to create a binary variable ("Once a month", "Once a week or more", and "Daily" coded in to "Yes", "Never" and "Rarely" coded in to "No").

Internalizing, externalizing and prosocial behavior at ages 3, 5, 7, 11 and 14 years were assessed by the Strengths and Difficulties Questionnaire (SDQ) (Goodman, 2001), completed by the parent, mainly mothers (more than $95 \%$ ). The SDQ is a screening questionnaire with extensive psychometric support (www.sdqinfo.com). In the MCS, construct, convergent, discriminant, and predictive validity have been established for the SDQ subscales at ages 3, 5 and 7 years, showing good internal reliability with alphas ranging from 0.66 to 0.82 for all subscales (Croft, Stride, Maughan, \& Rowe, 2015). The SDQ assesses internalizing behaviors in the past 6 months using ten items. Five from the "peer problems subscale" ("Generally plays alone", "Has one or more good friends", "Generally liked by other people", "Bullied or picked up by other children", "Gets on better with adults"), and five from the "emotional problems" subscale ("Gets a lot of headaches, stomach aches or sickness", "Worries a lot", "Often unhappy, down-hearted or tearful", "Nervous in new situations, easily loses confidence", "Has many fears, is easily scared").

The questionnaire assesses externalizing behaviors in the past 6 months using ten items. Five from the "conduct problems" subscale ("Often has temper tantrums or hot tempers", "Generally obedient, usually does what adults request", "Often fights with other children or bullies them", "Often lies or cheats", and "Steals from home, school or elsewhere"), and five from the "hyperactivity subscale" ("Is restless, cannot stay still for long", "Constantly fidgeting or squirming", "Easily distracted, finds it difficult to concentrate", "Things before doing things", "Finishes the work is doing, attention is good").

The questionnaire assesses prosocial behaviors in the past 6 months using five items ("Considerate to other people's feelings", "Shares readily with other children, for example toys, treats, pencils", "Helpful if someone is hurt, upset, or feeling ill", "Kind to younger children", "Often volunteers to help others"). Respondents have three options for each item ("Not true", "Somewhat true", and "Certainly true", coded into "0", "1", and "2" respectively) which were added to create a sum score for the three scales described above.

Covariates: We included gender as a potential effect modifier in our analyses. Additional covariates were the cohort child's ethnicity and poverty. Ethnicity was coded into "White", "Indian", "Pakistani and Bangladeshi", "Black or Black British", "Other (including Chinese)". For poverty we used a binary variable collected at age 9 months where a participant could fall either above or below the $60 \%$ median of the McClements equivalence scale (McClements, 1977). Ethnicity is associated with both mental health problems and childhood adversities, not due to biological differences because people from ethnic minority groups experience individual and societal barriers and disadvantages related to their ethnicity, including racism (O'Connor et al., 2020). Poverty has previously been shown to be a strong predictor of ACEs in the UK and to be related to poorer mental health in early life (R. E. Lacey, Howe, Kelly-Irving, Bartley, \& Kelly, 2020).

\subsection{Analyses}

The ACE scores were created by summing the binary values for each individual ACE to create a total score. This total score was collapsed to the following categories: 0 ACEs, 1 ACE, 2 ACEs and 3+ ACEs, consistent with many previous studies. In order to derive the ACEs clusters, latent class analysis was applied using the robust maximum likelihood estimator. We compared models for 2-6 classes. The best fitting class solution was determined by the model fit indices - Akaike's Information Criterion (AIC), Bayesian Information Criteria (BIC) and the sample-size adjusted Bayesian Information Criteria (SSABIC). We also inspected the entropy values. An entropy value approaching 1 suggests greater distinction of classes, and lower values of the AIC, BIC and SSABIC suggest a better fitting model.

In order to test associations between ACE scores, individual ACEs, LCA-derived ACEs clusters and our three outcomes of internalizing, externalizing and prosocial behaviors, we employed mixed effects linear regression models. The intercept was centred at age 3 (the first SDQ measurement) and the trajectories modelled through five waves of data to age 14. The internalizing, externalizing and prosocial domain scores were log-transformed for the analyses due to positive skew. The best fitting models (determined by comparing AIC and BIC values) were random intercepts and slopes with the inclusion of an interaction term between age and each ACEs variable plus an age-squared term. We applied the maximum likelihood estimator which included individuals in the analysis if they had at least one observation of internalizing $(n=8,855)$, externalizing $(n=8,862)$ or prosocial $(n=8,860)$ behaviors observed at any time point. 
Table 1

Descriptive statistics of the study sample.

\begin{tabular}{|c|c|c|c|c|c|c|}
\hline & $\mathrm{Mn}$ & M \% & $\mathrm{Fn}$ & $\mathrm{F} \%$ & Tot $n$ & Tot $\%$ \\
\hline \multicolumn{7}{|l|}{ ACEs } \\
\hline Parental separation NO & 4,224 & 93 & 4,054 & 93.7 & 8,278 & 93.4 \\
\hline YES & 315 & 7 & 274 & 6.3 & 589 & 6.6 \\
\hline Parental depression NO & 2,861 & 63 & 2,720 & 63 & 5,581 & 63 \\
\hline YES & 1,678 & 37 & 1,608 & 37 & 3,286 & 37 \\
\hline Parental drug use NO & 4,144 & 91.3 & 3,959 & 91.5 & 8,103 & 91.4 \\
\hline YES & 395 & 8.7 & 369 & 8.5 & 764 & 8.6 \\
\hline Parental alcohol use NO & 3,855 & 84.9 & 3,670 & 84.8 & 7,525 & 84.9 \\
\hline YES & 684 & 15.1 & 658 & 15.2 & 1,342 & 15.1 \\
\hline Inter-parent. use of force NO & 3,818 & 84 & 3,636 & 84 & 7,454 & 84 \\
\hline YES & 721 & 16 & 692 & 16 & 1,413 & 16 \\
\hline Parental discord NO & 3,814 & 84 & 3,623 & 84 & 7,437 & 84 \\
\hline YES & 725 & 16 & 705 & 16 & 1,430 & 16 \\
\hline Harsh parenting NO & 3,193 & 70 & 3,244 & 75 & 6,437 & 73 \\
\hline YES & 1,346 & 30 & 1,084 & 25 & 2,430 & 27 \\
\hline Physical punishment NO & 3,744 & 82.5 & 3,812 & 88 & 7,556 & 85 \\
\hline YES & 795 & 17.5 & 516 & 12 & 1,311 & 15 \\
\hline \multicolumn{7}{|l|}{ ACE score } \\
\hline 0 & 1,166 & 25.69 & 1,182 & 27.31 & 2,348 & 26.48 \\
\hline 1 & 1,438 & 31.68 & 1,461 & 33.7 & 2,899 & 32.69 \\
\hline 2 & 1,065 & 23.46 & 966 & 22.3 & 2,031 & 22.91 \\
\hline $3+$ & 870 & 19.17 & 719 & 16.6 & 1,589 & 17.92 \\
\hline SDQ & M (SD) (non log) & & $\mathrm{M}(\mathrm{SD})$ (non $\log$ ) & & M (SD) (non log) & \\
\hline int3 & $2.7(2.4)$ & & $2.5(2.3)$ & & $2.6(2.4)$ & \\
\hline int5 & $2.2(2.3)$ & & $2.2(2.3)$ & & $2.2(2.3)$ & \\
\hline int7 & $2.4(2.6)$ & & $2.3(2.4)$ & & $2.4(2.6)$ & \\
\hline int11 & $2.9(3.0)$ & & $2.9(2.9)$ & & $2.9(3.0)$ & \\
\hline int14 & $3.2(3.2)$ & & $3.6(3.2)$ & & $3.4(3.3)$ & \\
\hline ext3 & $6.7(3.7)$ & & $5.8(3.5)$ & & $6.3(3.6)$ & \\
\hline ext5 & $4.8(3.4)$ & & $3.8(3.0)$ & & $4.3(3.2)$ & \\
\hline ext7 & $4.9(3.5)$ & & $3.7(3.1)$ & & $4.3(3.4)$ & \\
\hline ext11 & $4.7(3.6)$ & & $3.5(3.4)$ & & $4.1(3.4)$ & \\
\hline ext14 & $4.5(3.5)$ & & $3.4(3.1)$ & & $3.9(3.3)$ & \\
\hline prosoc3 & 7.1 (1.9) & & $7.6(1.8)$ & & $7.3(1.8)$ & \\
\hline prosoc5 & $8.2(1.7)$ & & $8.7(1.5)$ & & $8.4(1.6)$ & \\
\hline prosoc7 & $8.3(1.7)$ & & $8.9(1.4)$ & & $8.6(1.6)$ & \\
\hline prosoc11 & $8.6(1.6)$ & & $9.1(1.2)$ & & $8.8(1.5)$ & \\
\hline prosoc14 & $8.1(1.9)$ & & $8.7(1.6)$ & & $8.4(1.8)$ & \\
\hline \multicolumn{7}{|l|}{ POVERTY } \\
\hline Above $60 \%$ median & 3,626 & 79.9 & 3,462 & 80.0 & 7,088 & 80.0 \\
\hline Below $60 \%$ median & 913 & 20.1 & 866 & 20.0 & 1,779 & 20.0 \\
\hline \multicolumn{7}{|l|}{ ETHNICITY } \\
\hline White & 4,178 & 92.3 & 3,954 & 91.5 & 8,132 & 91.9 \\
\hline Mixed & 87 & 1.9 & 69 & 1.6 & 156 & 1.8 \\
\hline Indian & 112 & 2.5 & 131 & 3.0 & 243 & 2.7 \\
\hline Pakistani \& Bangladeshi & 48 & 1.1 & 41 & 0.9 & 89 & 1.0 \\
\hline Black or Black British & 102 & 2.2 & 125 & 3.0 & 227 & 2.6 \\
\hline
\end{tabular}

NB Descriptive statistics presented for those with at least one outcome observed $(n=8,847)$.

In the first stage of the regression analyses we tested the associations between ACE operationalisation (ACE score, individual ACEs and LCA-derived ACE clusters) with each outcome in an unadjusted model. We then tested interactions between gender and ACEs. In the final stage we ran adjusted models including our covariates.

All analysis we run using STATA (StataCorp, 2017). The LCA model was implemented in Mplus (Muthén \& Muthén, 2018).

\section{Results}

The vast majority of participants were white (91.9\%), with $20 \%$ of all participants living in relative poverty. $33.1 \%$ of children experienced no ACEs by 3 years, $32.2 \%$ reported 1 ACE, $18.4 \%$ reported 2 ACEs and $16.3 \%$ reported 3 or more ACEs with no apparent differences between males and females. The most commonly reported ACEs were parental depression (40.0 \%), harsh parenting (27.1 $\%)$, physical punishment (16.2\%), inter-parental use of force (16.0\%) and parental alcohol misuse (13.7 \%). Harsh parenting and physical punishment seemed to affect more males than females, in both cases with a difference in prevalence of about $5 \%$. As expected, the average score for externalizing problems was higher for males and the average score for prosocial behavior was higher for females (Table 1). 
Table 2

Longitudinal associations between ACEs score (at 9 months and 3 years) and Internalizing, Externalizing, and Prosocial behaviors (from 3 to 14 years old).

\begin{tabular}{|c|c|c|c|c|c|c|c|c|c|c|c|c|}
\hline \multirow[b]{3}{*}{ ACE score } & \multicolumn{4}{|c|}{ Internalizing $(\mathrm{N}=8,571)$} & \multicolumn{4}{|c|}{ Externalizing $(\mathrm{N}=8,823)$} & \multicolumn{4}{|c|}{ Prosocial $(\mathrm{N}=8,859)$} \\
\hline & \multicolumn{2}{|c|}{ Non-adjusted } & \multicolumn{2}{|c|}{ Adjusted* } & \multicolumn{2}{|c|}{ Non-adjusted } & \multicolumn{2}{|c|}{ Adjusted* } & \multicolumn{2}{|c|}{ Non-adjusted } & \multicolumn{2}{|c|}{ Adjusted* } \\
\hline & $\log \mathrm{INT}$ & $95 \% \mathrm{CI}$ & $\log I N T$ & $95 \% \mathrm{CI}$ & $\log \mathrm{EXT}$ & $95 \% \mathrm{CI}$ & $\log \mathrm{EXT}$ & $95 \% \mathrm{CI}$ & $\log P R O$ & $95 \% \mathrm{CI}$ & $\log \mathrm{PRO}$ & $95 \% \mathrm{CI}$ \\
\hline 0 ACEs & Ref & & Ref & & Ref & & Ref & & Ref & & Ref & \\
\hline $1 \mathrm{ACE}$ & 0.056 & $0.021,0.092$ & 0.057 & $0.022,0.092$ & 0.144 & $0.110,0.177$ & 0.142 & $0.109,0.175$ & -0.020 & $-0.033,-0.007$ & -0.019 & $-0.032,-0.007$ \\
\hline 2 ACEs & 0.108 & $0.069,0.147$ & 0.108 & $0.070,0.146$ & 0.308 & $0.271,0.345$ & 0.299 & $0.263,0.335$ & -0.045 & $-0.059,-0.031$ & -0.042 & $-0.056,-0.028$ \\
\hline 3 ACEs & 0.211 & $0.170,0.252$ & 0.202 & $0.161,0.242$ & 0.441 & $0.402,0.480$ & 0.415 & $0.376,0.453$ & -0.065 & $-0.081,-0.050$ & -0.059 & $-0.073,-0.044$ \\
\hline Time/Slope & -0.022 & $-0.037,-0.023$ & -0.029 & $-0.036,-0.022$ & -0.115 & $-0.121,-0.109$ & -0.115 & $-0.121,-0.109$ & 0.063 & $0.060,0.064$ & 0.063 & $0.060,0.064$ \\
\hline 0 ACEs*time & Ref & & Ref & & Ref & & Ref & & Ref & & Ref & \\
\hline 1 ACE*time & 0.005 & $0.001,0.010$ & 0.006 & $0.001,0.010$ & -0.003 & $-0.007,0.001$ & -0.003 & $-0.007,0.002$ & 0.001 & $-0.001,0.002$ & 0.001 & $-0.001,0.002$ \\
\hline 2 ACEs*time & 0.011 & $0.005,0.016$ & 0.011 & $0.005,0.017$ & -0.005 & $-0.009,0.001$ & -0.005 & $-0.009,-0.001$ & 0.001 & $-0.001,0.003$ & 0.001 & $-0.001,0.003$ \\
\hline 3 ACEs*time & 0.008 & $0.002,0.014$ & 0.008 & $0.002,0.014$ & -0.004 & $-0.009,0.001$ & -0.004 & $-0.009,0.001$ & -0.001 & $-0.001,0.001$ & -0.001 & $-0.002,0.002$ \\
\hline Time-squared & 0.004 & $0.003,0.004$ & 0.004 & $0.003,0.004$ & 0.007 & $0.007,0.008$ & 0.007 & $0.007,0.008$ & -0.004 & $-0.005,-0.004$ & -0.004 & $-0.005,-0.004$ \\
\hline Intercept & 0.797 & $0.769,0.824$ & 0.751 & $0.721,0.781$ & 1.420 & $1.369,1.446$ & 1.473 & $1.445,1.502$ & 1.999 & $1.988,2.012$ & 1.970 & $1.960,1.981$ \\
\hline
\end{tabular}

*Adjusted models include gender, ethnicity, and poverty. 


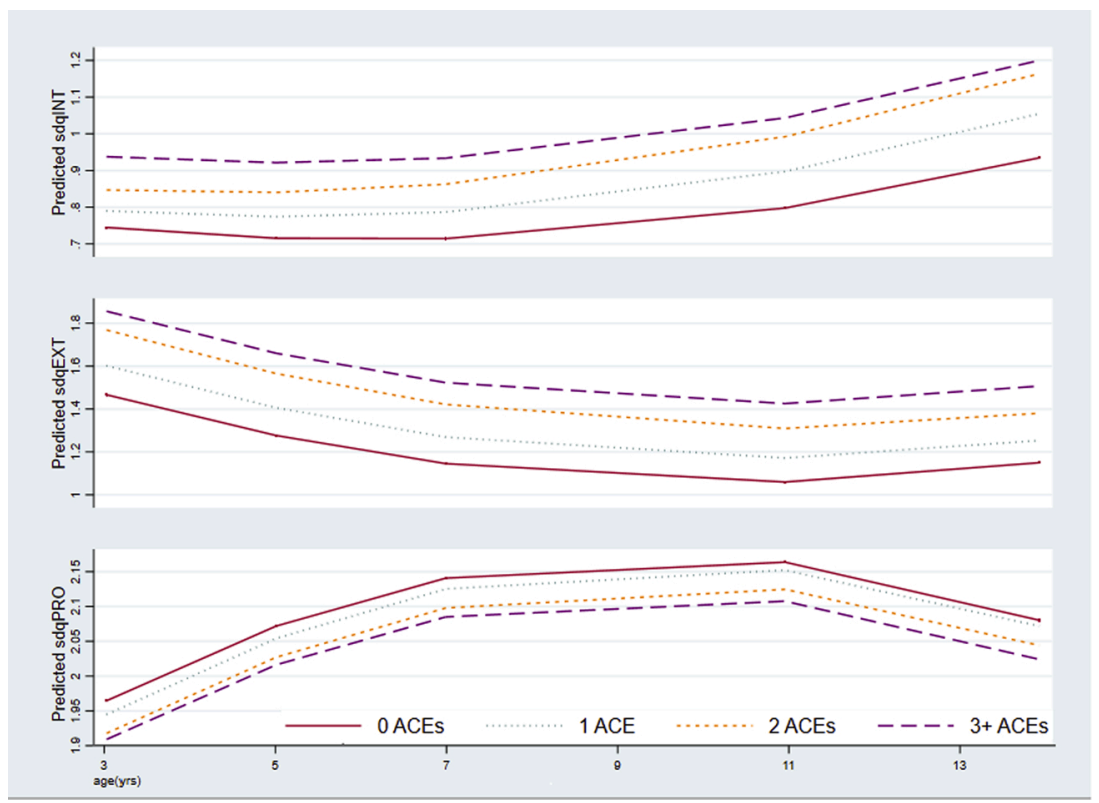

Fig. 1. Internalizing, externalizing, and prosocial behavior (indicated by logSDQ scores on the $\mathrm{Y}$ axis) from 3 to 14 in participants experiencing 0 , 1 , 2 , and $3+$ ACEs.

\subsection{Associations between ACE score and SDQ scores}

We did not find any gender differences in associations between any of our ACE operationalisations and outcomes. Therefore we henceforth present all regression results for boys and girls combined.

On average, internalizing problems increase between ages 3-14 years. There was a graded positive association between the number of ACEs and the number of internalizing symptoms reported at age 3 (Table 2, Fig. 1), which changed very little upon inclusion of covariates. These differences remained through to age 14 but there was evidence of divergence between those reporting 0 and 2 ACEs over time. However, stark associations remained between the ACEs score reported by age 3 and internalizing behaviors by age 14 .

On average, externalizing problems declined over time through to age 11 but then increased by age 14. Similar to internalizing problems, there was a graded association between the number of ACEs reported and externalizing symptoms at age 3 (Table 2, Fig. 1). These differences remained through to age 14 and there was suggestion that those reporting 2 ACEs had a flatter slope over time compared to those reporting no ACEs. Again these results remained upon adjustment for poverty and ethnicity.

On average, prosocial behaviors increased over time through to age 11 but then declined by age 14. Again, there was a graded association between the number of ACEs reported and prosocial score at age 3 (more ACEs, lower score). We did not observe differences in the rate of change over time, and thus there remained a graded pattern in the trajectories through to age 14.

\subsection{Associations between individual ACEs and SDQ scores}

Parental separation, parental depression, parental drug use, parental discord, harsh parenting, inter-parental use of force, and physical punishment were associated with higher scores on the internalizing problems subscale by age 3 in the unadjusted and adjusted models (Table 3). For parental separation and parental depression the trajectories of internalizing symptoms increased over time relative to children not experiencing either of these two ACEs. For parental discord, harsh parenting, parental drug use and physical punishment, the trajectories of internalizing problems remained at a heightened level through to age 14 and the rate of change did not vary for these ACEs. Parental alcohol misuse was not associated with differences in internalizing symptom trajectories in this study by age 3 or the rate of change over time. The most marked associations for internalizing problems were observed for children who had parents with parental discord and who had a depressed parent between 9 months and 3 years compared to those who did not (Fig. 2).

For externalizing symptoms, all of the ACEs considered were associated with higher externalizing problems scores at age 3 in both the unadjusted and adjusted models (Table 3). For those who experienced parental separation and inter-parental use of force the rate of change increased over time. For harsh parenting and parental alcohol misuse the decrease in externalizing problems over time was more marked, suggesting that this association is stronger at earlier stages of life. For harsh parenting, parental depression, parental alcohol use, parental drug use and physical punishment, the trajectories of externalizing problems remained at a higher level through to age 14 relative to those who did not report these ACEs. The most marked differences for what concerns externalizing problems were observed between those children who experienced harsh parenting and physical punishment by age 3 years compared to those who did not (Fig. 2).

For prosocial symptoms (Table 3), children who experienced parental depression, parental alcohol misuse, parental discord, harsh 
Table 3

Longitudinal associations between individual ACEs (at 9 months and 3 years) and Internalizing, Externalizing, and Prosocial behaviors (from 3 to 14 years old).

\begin{tabular}{|c|c|c|c|c|c|c|}
\hline & \multicolumn{2}{|c|}{ Internalising $\log (\mathrm{N}=8571)$} & \multicolumn{2}{|c|}{ Externalising $\log (\mathrm{N}=8823)$} & \multicolumn{2}{|c|}{ Prosocial $\log (\mathrm{N}=8859)$} \\
\hline & $\log$ INT & $95 \%$ CI & $\log \mathrm{EXT}$ & $95 \%$ CI & $\log \mathrm{PRO}$ & $95 \%$ CI \\
\hline Parental separation & 0.059 & $0.005,0.112$ & 0.166 & $0.114,0.219$ & 0.007 & $-0.012,0.026$ \\
\hline Slope & -0.024 & $-0.030,-0.018$ & -0.114 & $-0.120,-0.107$ & 0.063 & $0.061,0.064$ \\
\hline Parental separation*time & 0.014 & $0.006,0.022$ & 0.009 & $0.002,0.017$ & -0.005 & $-0.009,-0.002$ \\
\hline Time-squared & 0.004 & $0.003,0.004$ & 0.007 & $0.006,0.007$ & -0.004 & $-0.005,-0.004$ \\
\hline Intercept & 0.830 & $0.811,0.850$ & 1.658 & $1.637,1.679$ & 1.942 & $1.936,1.949$ \\
\hline Parental depression & 0.113 & $0.078,0.142$ & 0.165 & $0.139,0.192$ & -0.016 & $-0.026,-0.006$ \\
\hline Slope & -0.028 & $-0.031,-0.014$ & -0.118 & $-0.123,-0.113$ & 0.062 & $0.060,0.064$ \\
\hline Parental depression*time & 0.011 & $0.007,0.015$ & 0.001 & $-0.002,0.005$ & 0.001 & $-0.001,0.002$ \\
\hline Time-squared & 0.004 & $0.003,0.004$ & 0.007 & $0.006,0.007$ & -0.004 & $-0.005,-0.004$ \\
\hline Intercept & 0.791 & $0.766,0.816$ & 1.606 & $1.585,1.628$ & 1.941 & $1.932,1.950$ \\
\hline Parental drug use & 0.072 & $0.024,0.119$ & 0.120 & $0.074,0.166$ & 0.002 & $-0.014,0.019$ \\
\hline Slope & -0.023 & $-0.029,-0.017$ & -0.118 & $-0.123,-0.113$ & 0.064 & $0.062,0.067$ \\
\hline Parental drug use*time & -0.001 & $-0.007,0.006$ & 0.002 & $-0.003,0.008$ & -0.004 & $-0.007,-0.001$ \\
\hline Time-squared & 0.004 & $0.003,0.004$ & 0.007 & $0.006,0.007$ & -0.004 & $-0.005,-0.004$ \\
\hline Intercept & 0.827 & $0.807,0.847$ & 1.658 & $1.637,1.680$ & 1.943 & $1.936,1.950$ \\
\hline Parental alcohol misuse & 0.018 & $-0.018,0.055$ & 0.048 & $0.012,0.084$ & -0.015 & $-0.028,-0.002$ \\
\hline Slope & -0.023 & $-0.026,-0.009$ & -0.117 & $-0.122,-0.112$ & 0.064 & $0.061,0.066$ \\
\hline Parental alcohol use ${ }^{*}$ time & -0.001 & $-0.006,0.004$ & -0.004 & $-0.008,-0.001$ & 0.002 & $0.001,0.004$ \\
\hline Time-squared & 0.004 & $0.003,0.004$ & 0.007 & $0.006,0.007$ & -0.004 & $-0.005,-0.004$ \\
\hline Intercept & 0.830 & $0.809,0.850$ & 1.657 & 1.637, 1.677 & 1.945 & $1.938,1.953$ \\
\hline Inter-parental use of force & 0.046 & $0.010,0.082$ & 0.097 & $0.062,0.132$ & -0.011 & $-0.025,0.013$ \\
\hline Slope & -0.024 & $-0.030,-0.018$ & -0.119 & $-0.124,-0.114$ & 0.064 & $0.061,0.067$ \\
\hline Inter-parental use of force*time & 0.006 & $-0.001,0.011$ & 0.007 & $0.002,0.012$ & -0.001 & $-0.003,0.001$ \\
\hline Time-squared & 0.004 & $0.003,0.004$ & 0.007 & $0.006,0.007$ & -0.004 & $-0.005,-0.004$ \\
\hline Intercept & 0.826 & $0.806,0.846$ & 1.650 & $1.630,1.670$ & 1.945 & $1.938,1.952$ \\
\hline Parental discord & 0.125 & $0.089,0.161$ & 0.150 & $0.115,0.185$ & -0.028 & $-0.041,-0.015$ \\
\hline Slope & -0.024 & $-0.026,-0.008$ & -0.118 & $-0.123,-0.113$ & 0.064 & $0.061,0.066$ \\
\hline Parental discord time & 0.004 & $-0.001,0.010$ & 0.003 & $-0.001,0.008$ & -0.002 & $-0.003,0.001$ \\
\hline Time-squared & 0.003 & $0.002,0.004$ & 0.007 & $0.006,0.008$ & -0.004 & $-0.005,-0.004$ \\
\hline Intercept & 0.815 & $0.795,0.835$ & 1.644 & $1.624,1.663$ & 1.947 & $1.940,1.954$ \\
\hline Harsh parenting & 0.073 & $0.038,0.109$ & 0.270 & $0.241,0.298$ & -0.046 & $-0.057,-0.035$ \\
\hline Slope & -0.022 & $-0.028,-0.016$ & -0.114 & $-0.119,-0.109$ & 0.062 & $0.060,0.064$ \\
\hline Harsh parenting*time & -0.002 & $-0.007,0.002$ & -0.011 & $-0.015,-0.007$ & 0.001 & $-0.001,0.001$ \\
\hline Time-squared & 0.003 & $0.002,0.004$ & 0.007 & $0.006,0.007$ & -0.004 & $-0.005,-0.004$ \\
\hline Intercept & 0.809 & $0.788,0.831$ & 1.580 & $1.560,1.601$ & 1.958 & $1.950,1.965$ \\
\hline Physical punishment & 0.096 & $0.058,0.133$ & 0.256 & $0.220,0.292$ & -0.058 & $-0.071,-0.044$ \\
\hline Slope & -0.023 & $-0.029,-0.016$ & -0.113 & $-0.119,-0.106$ & 0.062 & $0.060,0.064$ \\
\hline Physical punishment*time & -0.001 & $-0.006,0.004$ & -0.002 & $-0.006,0.002$ & 0.001 & $-0.001,0.002$ \\
\hline Time-squared & 0.003 & $0.003,0.004$ & 0.007 & $0.006,0.008$ & -0.004 & $-0.005,-0.004$ \\
\hline Intercept & 0.816 & $0.795,0.836$ & 1.617 & $1.595,1.639$ & 1.953 & $1.946,1.960$ \\
\hline
\end{tabular}

" Adjusted models include gender, ethnicity, and poverty.

parenting and physical punishment had a lower prosocial score by age 3 . Those experiencing parental separation showed no differences in prosocial scores by age 3 but had a flatter slope over time relative to their peers who did not experience this ACE. Those experiencing parental alcohol use had a lower prosocial score at age 3 and a slightly steeper increase through to age 14 . Children who had parents who were drug users had similar prosocial scores as their peers without this ACE but a flatter increase through to age 14 . The most marked differences for prosocial behaviors were observed between those children who experienced being physically punished and harsh parenting and those who did not.

\subsection{Application of LCA to ACEs data}

The LCA did not work in this dataset - the entropy level was very low $\sim 0.3-0.4$, suggesting that it could not discriminate between different groups of ACEs reporting. Hence, we were unable to analyse associations for LCA-derived clusters in this study.

\section{Discussion}

In this study we explored the association between different ways of operationalising ACEs and later trajectories of internalizing, externalizing, and prosocial behavior from age 3-14. We employed three approaches to operationalising ACEs - ACE scores, individual ACEs, and ACEs clustering using latent class analysis. In line with our first hypothesis, we found that a higher number of ACEs predicted worse mental health and prosocial outcomes in childhood and adolescence. In most cases these differences were evident by age 3 and persisted through to adolescence. The associations with ACE scores showed a dose-response pattern, with better outcomes for 


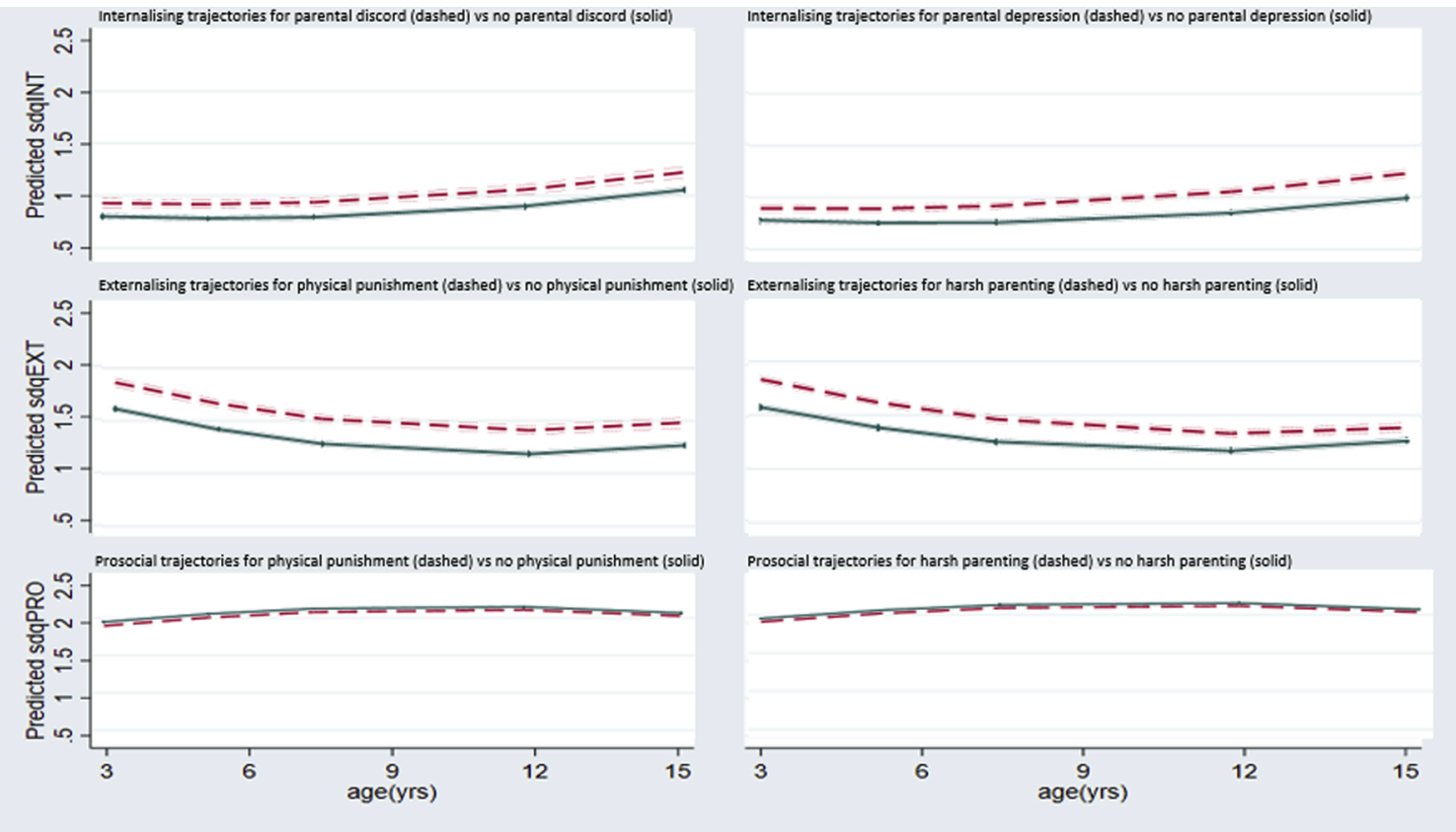

Fig. 2. Internalizing, externalizing, and prosocial behavior (indicated by logSDQ scores on the $\mathrm{Y}$ axis) from 3 to 14 in participants experiencing ACEs (here showing the two ACEs with the highest coefficients only for each outcome).

those experiencing no ACEs, and the poorest outcomes for those experiencing 3 or more ACEs. This finding is in line with the majority of the literature in this field which looked at the cumulative effects of ACEs on mental health outcomes (Chapman et al., 2004; Felitti et al., 1998) but we extended this work to look at differences in mental health from early childhood, as well as including prosocial and negative mental health outcomes. Despite the well-known limitations with the ACE score approach (Rebecca E Lacey \& Minnis, 2020), ACE scores were neatly associated with internalizing, externalizing and prosocial behavior in this study.

In line with our second hypothesis, ACEs which appear to produce particularly stark differences in terms of early mental health outcomes were physical punishment and harsh parenting (particularly for externalizing behaviors), but also parental discord and parental depression (particularly for internalizing problems). Previous research has pointed out how harsh forms of discipline and physical forms of abuse in particular can have long-lasting impacts on future behavioral and mental health (Farrington, 2005; Fergusson, Boden, \& Horwood, 2008; Gershoff \& Grogan-Kaylor, 2016; Gross \& Keller, 1992). Several mechanisms have been proposed including children emulating aggressive behavior modelled by parents (Gershoff \& Bitensky, 2007), as well as long-term damage to the HPA axis caused by threat-related stressors such as harsh parenting and physical abuse (Tomoda et al., 2009). Also, there is substantial evidence that this damage can result in reduced behavioral and emotion regulation (Kliewer, 2016).

The ACEs that showed the strongest impact on internalizing problems were parental discord and parental depression. Some researchers have suggested that the association between parental discord and internalizing symptoms in childhood and adolescence may be explained by attributions of self-blame or feelings of guilt in the child (Cummings, Davies, \& Campbell, 2002; Grych, Fincham, Jouriles, \& McDonald, 2000). This association between parental depression and internalizing problems in children may also be partly explained by the interaction between genetic factors, such as the transmission of genetic vulnerability to experience depressive symptomatology from parent to child, and also by factors that are related to the relational aspects of the parent child dyad (Silberg, Maes, \& Eaves, 2010). For example, previous research suggested that depressed parents are less likely to have warm and affectionate interactions with their children (Jacob \& Johnson, 1997). The interplay between these factors may contribute to the development of internalizing symptoms in the child as early as 3 years, and throughout adolescence.

We touched on emulation of parental behavior as a potential mechanism that underlies the association between harsh discipline and externalising behaviours. We argue that this may be true for internalizing behaviors as well, and particularly important for prosocial behaviors. In fact, several studies suggest that the development of prosocial behaviours rely significantly on imitation mechanisms and that infants prefer prosocial over antisocial behaviour (Hamlin \& Wynn, 2011; Williamson, Donohue, \& Tully, 2013).

In general, we observed that the effects of ACEs were stronger for externalizing behaviors, compared to internalizing and prosocial behaviors, as we have seen in both unadjusted and adjusted models. It could be that by 'acting out', children manage to relieve their stress and discomfort more effectively. In addition, it could be that externalizing problems are a more likely outcome due to the fact that the neural substrates that underlie self-control are not yet fully developed in children and young people, and as such this might be a more 'developmentally appropriate' response during this life stage (E. K. Miller \& Cohen, 2001).

In our third hypothesis we thought that ACEs would cluster in the MCS in line with previous studies applying LCA (R. E. Lacey et al., 2020). However the LCA suggested that the ACEs considered here did not cluster in the MCS. This was unexpected and may suggest 
that the timing of ACEs in this particular study ( 9 months and 3 years) may have been too early to allow for any accumulation of ACEs in order to detect their clustering. Future studies might investigate the correlation between ACEs over time. Also, it could be that respondent bias (information about ACEs were collected from mothers and fathers) resulted in under-reporting of ACEs, with a consequent lack of sufficient information to allow the clustering to emerge. However, we argue that our results may reflect an actual lack of significant clustering at the level of the specific ACEs we considered. This is supported by a number of factors such as the large sample size, the stringent criteria we applied to determine whether an individual would classify as having experienced an ACE or not, and a sensitivity analysis run for ACEs separately for 9 months and 3 years, which yielded even lower correlation coefficients than the ACEs considered by collapsing information at 9 months and 3 years together (see Tables A2 and A3 in the Appendix A).

Finally, we anticipated that associations would differ for girls and boys, in line with previous research (Edwards, Holden, Felitti, \& Anda, 2003). However, none of the gender*ACE interactions were significant suggesting that in this sample the associations were similar for girls and boys. Given our large sample size, it is unlikely that this result is the consequence of lacking statistical power in our analyses. Although previous research has indicated that females may be more vulnerable to ACEs when looking at long term effects (Haatainen et al., 2003; Meng \& D'Arcy, 2016), we only found some evidence for a difference in prevalence of harsh parenting and physical punishment, with more males suffering from these ACEs. Our results suggest that at least in the MCS, the effect of the ACEs we considered may be relatively similar for males and females. Future studies can shed light on this by trying to operationalize ACEs in different ways and conduct multiple analysis to test whether results hold for males and females across the different operationalization methods, and also by looking at more distal outcomes to see if gender differences emerge later on.

\subsection{Strengths and limitations}

The present work has several strengths, including the use of a large nationally representative sample and the use of multiple outcome measures completed by parents at 5 time points from childhood to adolescence. We also had ACEs prospectively collected from two time points in very early childhood ( 9 months and 3 years) from both parents. As such, this is one of the first studies to demonstrate the immediate and also persistent effects of ACEs experienced during very early childhood. We not only focused on externalizing and internalizing behaviors, but also on prosocial behaviors - a frequently overlooked domain of child mental health development.

We highlight several limitations including potential reporting bias at the level of the ACEs, and the lack of information regarding other frequently researched adversities such as sexual abuse, racial discrimination, and antisocial behavior in the family. In addition, both our parental discord and harsh parenting variables consist of some but not all items present in the original scales (GRIMS and Straus Conflict Scale), although we demonstrated excellent levels of construct validity with the items that were available to us. Although we employed a complete-case analysis strategy, we used maximum likelihood estimation in the modelling strategy. This allowed us to include all participants with at least one data point at the level of the outcomes.

\subsection{Conclusions and future directions}

The present study has highlighted that ACEs scores and individual ACEs are associated with later internalizing, externalizing, and prosocial behaviors, from childhood to adolescence. There appears to be no particular clustering of ACEs in the MCS dataset and associations did not vary for girls and boys. These findings have important clinical and policy implications. They suggest that taking into account not only the number of stressors that individuals are exposed to from very early age, but also the specific type of stressors, is helpful in predicting an individual's mental health development, and thus implement appropriate intervention programmes. With respect to specific ACEs showing the most pervasive influences on mental health problems in this study, our findings lend further support to existing calls from advocates for the human rights and welfare of children to abolish physical punishment in all settings including the family (Organization, 2016). There is good evidence that legal bans are associated with accelerated declines in the prevalence of physical punishment as well as reductions in severe physical abuse (Zolotor \& Puzia, 2010). In the UK, legislative change to end physical punishment by parents has been enacted in 2019 in Scotland and in 2020 in Wales, but is yet to be introduced in England and Northern Ireland. At the same time, it is imperative to support parental mental health and partnerships in the early years of a child's life. Future studies could also focus on the timing, severity and chronicity of specific ACEs in order to better understand the impact of these on later mental health. We highlighted in the previous section that a potential limitation of the present study is the lack of multiple sources of information (i.e. we only had parent-reported data available). Future studies should try to make use of combined data sources (or preferring teacher-reported data over parent-reported or self-report when possible).

It would be particularly interesting to investigate whether there are specific stressors that predict internalizing symptoms over and above externalizing symptoms. In the present study, all ACEs showed a stronger association with externalizing rather than internalizing behaviors, even when we looked at parental depression, which may be hypothesised to predict internalizing symptoms particularly well. Future research should also investigate the causal mechanisms that underlie the associations between ACEs and later mental health and behavioral problems. Within the broader field of life course epidemiology, this can be partially done by implementing causal models (i.e. G-methods). However, we advocate for a dialogue between multiple disciplines, including clinical and developmental psychology, neurobiology, and epidemiology to disentangle the causal mechanisms that underlie the relationship between ACEs and later mental health difficulties. Understanding these mechanisms will help pave the way towards designing and delivering targeted prevention and intervention programmes for at-risk youth. 


\section{Funding}

LB and RL are supported by the Economic and Social Research Council [grant number: ES/P010229/1].

\section{Declaration of Competing Interest}

All authors declare that they have no conflict of interest

\section{Acknowledgements}

We are grateful for the Millennium Cohort Members and their families for their ongoing participation in the study. We also thank the UK Data Service for making these data available for researchers and thank the Centre for Longitudinal Studies at the UCL Institute of Education who runs the study.

\section{Appendix A}

Table A1

Table A1

Tetrachoric correlation matrix between ACEs included in our study observed at 9 months and/or 3 years.

\begin{tabular}{|c|c|c|c|c|c|c|c|c|}
\hline & $\begin{array}{l}\text { Parent } \\
\text { separation }\end{array}$ & $\begin{array}{l}\text { Parental } \\
\text { depression }\end{array}$ & $\begin{array}{l}\text { Parental } \\
\text { drug use }\end{array}$ & $\begin{array}{l}\text { Parental } \\
\text { alcohol use }\end{array}$ & $\begin{array}{l}\text { Inter-parental } \\
\text { use of force }\end{array}$ & $\begin{array}{l}\text { Parental } \\
\text { discord }\end{array}$ & $\begin{array}{l}\text { Harsh } \\
\text { parenting }\end{array}$ & $\begin{array}{l}\text { Smacking } \\
\text { child }\end{array}$ \\
\hline Parent separation & 1 & & & & & & & \\
\hline $\begin{array}{l}\text { Parental } \\
\text { depression }\end{array}$ & 0.245 & 1 & & & & & & \\
\hline Parental drug use & 0.039 & 0.196 & 1 & & & & & \\
\hline $\begin{array}{l}\text { Parental alcohol } \\
\text { use }\end{array}$ & -0.197 & 0.064 & 0.281 & 1 & & & & \\
\hline $\begin{array}{l}\text { Inter-parental use } \\
\text { of force }\end{array}$ & 0.093 & 0.229 & 0.325 & 0.193 & 1 & & & \\
\hline Parental discord & 0.308 & 0.298 & 0.185 & 0.094 & 0.397 & 1 & & \\
\hline Harsh parenting & -0.003 & 0.029 & 0.108 & 0.060 & 0.048 & 0.026 & 1 & \\
\hline Smacking child & -0.042 & 0.047 & -0.010 & -0.007 & 0.126 & 0.092 & 0.382 & 1 \\
\hline
\end{tabular}

Table A2

Tetrachoric correlation matrices between ACEs included in our study observed at 9 months (some ACEs were not available at this time point).

\begin{tabular}{llll}
\hline 9 months & Parental separation & Parental discord & Parental depression \\
\hline Parental separation & $\mathbf{1}$ & & \\
Parental discord & 0.28 & 0.16 & $\mathbf{1}$ \\
Parental depression & 0.07 & -0.18 & 0.05 \\
Parental alcohol use & 0.03 & $\mathbf{1}$ & \\
\hline
\end{tabular}

Table A3

Tetrachoric correlation matrices between ACEs included in our study observed at 3 years (some ACEs were not available at this time point).

\begin{tabular}{|c|c|c|c|c|c|c|}
\hline 3 years & Parental separation & Parental discord & Parental depression & Parental drug use & Harsh parenting & Parental alcohol use \\
\hline Parental separation & 1 & & & & & \\
\hline Parental discord & 0.23 & 1 & & & & \\
\hline Parental depression & 0.27 & 0.22 & 1 & & & \\
\hline Parental drug use & 0.20 & 0.38 & 0.20 & 1 & & \\
\hline Harsh parenting & -0.02 & 0.11 & 0.05 & 0.06 & 1 & \\
\hline Parental alcohol use & 0.04 & -0.13 & 0.06 & 0.09 & 0.03 & 1 \\
\hline
\end{tabular}

\section{References}

Anda, R. F., Felitti, V. J., Bremner, J. D., Walker, J. D., Whitfield, C., Perry, B. D., \& Giles, W. H. (2006). The enduring effects of abuse and related adverse experiences in childhood. European Archives of Psychiatry and Clinical Neuroscience, 256(3), 174-186.

Appleyard, K., Egeland, B., van Dulmen, M. H., \& Alan Sroufe, L. (2005). When more is not better: The role of cumulative risk in child behavior outcomes. Journal of Child Psychology and Psychiatry, 46(3), 235-245. 
Baglivio, M. T., Epps, N., Swartz, K., Huq, M. S., Sheer, A., \& Hardt, N. S. (2014). The prevalence of adverse childhood experiences (ACE) in the lives of juvenile offenders. Journal of Juvenile Justice, 3(2).

Bellis, M. A., Lowey, H., Leckenby, N., Hughes, K., \& Harrison, D. (2014). Adverse childhood experiences: retrospective study to determine their impact on adult health behaviours and health outcomes in a UK population. Journal of Public Health, 36(1), 81-91.

Borges, G., Angst, J., Nock, M. K., Ruscio, A. M., \& Kessler, R. C. (2008). Risk factors for the incidence and persistence of suicide-related outcomes: A 10-year follow-up study using the National Comorbidity Surveys. Journal of Affective Disorders, 105(1-3), 25-33. https://doi.org/10.1016/j.jad.2007.01.036

Carr, C. P., Martins, C. M., Stingel, A. M., Lemgruber, V. B., \& Juruena, M. F. (2013). The role of early life stress in adult psychiatric disorders: A systematic review according to childhood trauma subtypes. The Journal of Nervous and Mental Disease, 201(12), 1007-1020. https://doi.org/10.1097/nmd.0000000000000049

Chapman, D. P., Whitfield, C. L., Felitti, V. J., Dube, S. R., Edwards, V. J., \& Anda, R. F. (2004). Adverse childhood experiences and the risk of depressive disorders in adulthood. Journal of Affective Disorders, 82(2), 217-225.

Chen, M., \& Lacey, R. E. (2018). Adverse childhood experiences and adult inflammation: Findings from the 1958 British birth cohort. Brain, Behavior, and Immunity, $69,582-590$

Connelly, R., \& Platt, L. (2014). Cohort profile: UK millennium cohort study (MCS). International Journal of Epidemiology, 43(6), 1719-1725. https://doi.org/10.1093/ ije/dyu001

Croft, S., Stride, C., Maughan, B., \& Rowe, R. (2015). Validity of the strengths and difficulties questionnaire in preschool-aged children. Pediatrics, 135(5), e1210-e1219.

Cummings, E. M., Davies, P. T., \& Campbell, S. B. (2002). Developmental psychopathology and family process: Theory, research, and clinical implications. Guilford Press.

Cunningham, T. J., Ford, E. S., Croft, J. B., Merrick, M. T., Rolle, I. V., \& Giles, W. H. (2014). Sex-specific relationships between adverse childhood experiences and chronic obstructive pulmonary disease in five states. International Journal of Chronic Obstructive Pulmonary Disease, 9, 1033-1042. https://doi.org/10.2147/copd. S68226

Danese, A. (2020). Annual Research Review: Rethinking childhood trauma-new research directions for measurement, study design and analytical strategies. Journal of Child Psychology and Psychiatry, 61(3), 236-250.

Edwards, V. J., Holden, G. W., Felitti, V. J., \& Anda, R. F. (2003). Relationship between multiple forms of childhood maltreatment and adult mental health in community respondents: Results from the adverse childhood experiences study. The American Journal of Psychiatry, 160(8), 1453-1460.

Eisenberg, N., Fabes, R. A., \& Spinrad, T. (2006). Handbook of child psychology. John Wiley \& Sons.

Farrington, D. P. (2005). Childhood origins of antisocial behavior. Clinical Psychology \& Psychotherapy: An International Journal of Theory \& Practice, 12(3), 177-190.

Felitti, V. J., Anda, R. F., Nordenberg, D., Williamson, D. F., Spitz, A. M., Edwards, V., ... Marks, J. S. (1998). Relationship of childhood abuse and household dysfunction to many of the leading causes of death in adults: The Adverse Childhood Experiences (ACE) Study. American Journal of Preventive Medicine, 14(4), $245-258$.

Fergusson, D. M., Boden, J. M., \& Horwood, L. J. (2008). Exposure to childhood sexual and physical abuse and adjustment in early adulthood. Child Abuse \& Neglect, 32(6), 607-619.

Flouri, E., \& Panourgia, C. (2011). Adverse life events and emotional and behavioral problems in adolescence: The role of non-verbal cognitive ability and negative cognitive errors. Journal of Abnormal Child Psychology, 39(5), 695-709.

Fuller-Thomson, E., Filippelli, J., \& Lue-Crisostomo, C. A. (2013). Gender-specific association between childhood adversities and smoking in adulthood: findings from a population-based study. Public Health, 127(5), 449-460. https://doi.org/10.1016/j.puhe.2013.01.006

Gershoff, E. T., \& Bitensky, S. H. (2007). The case against corporal punishment of children: Converging evidence from social science research and international human rights law and implications for US public policy. Psychology Public Policy and Law, 13(4), 231.

Gershoff, E. T., \& Grogan-Kaylor, A. (2016). Spanking and child outcomes: Old controversies and new meta-analyses. Journal of Family Psychology, $30(4), 453$.

Goodday, S., Shuldiner, J., Bondy, S., \& Rhodes, A. (2019). Exposure to parental psychopathology and offspring's risk of suicide-related thoughts and behaviours: a systematic review. Epidemiology and Psychiatric Sciences, 28(2), 179-190.

Goodman, R. (2001). Psychometric properties of the strengths and difficulties questionnaire. Journal of the American Academy of Child and Adolescent Psychiatry, 40 (11), 1337-1345

Gross, A. B., \& Keller, H. R. (1992). Long-term consequences of childhood physical and psychological maltreatment. Aggressive Behavior, 18(3), 171-185.

Grych, J. H., Fincham, F. D., Jouriles, E. N., \& McDonald, R. (2000). Interparental conflict and child adjustment: Testing the mediational role of appraisals in the cognitive-contextual framework. Child Development, 71(6), 1648-1661.

Haatainen, K. M., Tanskanen, A., Kylmä, J., Honkalampi, K., Koivumaa-Honkanen, H., Hintikka, J., \& Viinamäki, H. (2003). Gender differences in the association of adult hopelessness with adverse childhood experiences. Social Psychiatry and Psychiatric Epidemiology, 38(1), 12-17.

Hailey Maier, E., \& Lachman, M. E. (2000). Consequences of early parental loss and separation for health and well-being in midlife. International Journal of Behavioral Development, 24(2), 183-189.

Hamlin, J. K., \& Wynn, K. (2011). Young infants prefer prosocial to antisocial others. Cognitive Development, 26(1), 30-39. https://doi.org/10.1016/j. cogdev.2010.09.001

Hengartner, M. P., Ajdacic-Gross, V., Rodgers, S., Muller, M., \& Rossler, W. (2013). Childhood adversity in association with personality disorder dimensions: new findings in an old debate. European Psychiatry: The Journal of the Association of European Psychiatrists, 28(8), 476-482. https://doi.org/10.1016/j. eurpsy.2013.04.004

Hillberg, T., Hamilton-Giachritsis, C., \& Dixon, L. (2011). Review of meta-analyses on the association between child sexual abuse and adult mental health difficulties: A systematic approach. Trauma, Violence \& Abuse, 12(1), 38-49.

Ho, G. W., Chan, A. C., Chien, W.-T., Bressington, D. T., \& Karatzias, T. (2019). Examining patterns of adversity in Chinese young adults using the Adverse Childhood Experiences-International Questionnaire (ACE-IQ). Child Abuse \& Neglect, 88, 179-188.

Hughes, K., Bellis, M. A., Hardcastle, K. A., Sethi, D., Butchart, A., Mikton, C., \& Dunne, M. P. (2017). The effect of multiple adverse childhood experiences on health: a systematic review and meta-analysis. The Lancet Public Health, 2(8), e356-e366.

Jacob, T., \& Johnson, S. L. (1997). Parent-Child interaction among depressed fathers and mothers: Impact on child functioning. Journal of Family Psychology, 11(4), 391.

Kalmakis, K. A., \& Chandler, G. E. (2015). Health consequences of adverse childhood experiences: A systematic review. Journal of the American Association of Nurse Practitioners, 27(8), 457-465. https://doi.org/10.1002/2327-6924.12215

Kliewer, W. (2016). Victimization and biological stress responses in urban adolescents: Emotion regulation as a moderator. Journal of Youth and Adolescence, 45(9), $1812-1823$.

Lacey, R. E., \& Minnis, H. (2020). Practitioner Review: Twenty years of research with adverse childhood experience scores-Advantages, disadvantages and applications to practice. Journal of Child Psychology and Psychiatry, 61(2), 116-130.

Lacey, R. E., Bartley, M., Pikhart, H., Stafford, M., \& Cable, N. (2014). Parental separation and adult psychological distress: An investigation of material and relational mechanisms. BMC Public Health, 14(1), 272. https://doi.org/10.1186/1471-2458-14-272

Lacey, R. E., Howe, L. D., Kelly-Irving, M., Bartley, M., \& Kelly, Y. (2020). The clustering of adverse childhood experiences in the avon longitudinal study of parents and children: Are gender and poverty important? Journal of Interpersonal Violence. https://doi.org/10.1177/0886260520935096, 886260520935096.

Luecken, L. J., \& Roubinov, D. S. (2012). Pathways to lifespan health following childhood parental death. Social and Personality Psychology Compass, 6(3), 243-257.

Mack, K. Y. (2001). Childhood family disruptions and adult well-being: The differential effects of divorce and parental death. Death Studies, 25 (5), 419-443.

Masten, A. S., Coatsworth, J. D., Neemann, J., Gest, S. D., Tellegen, A., \& Garmezy, N. (1995). The structure and coherence of competence from childhood through adolescence. Child Development, 66(6), 1635-1659.

Masyn, K. E. (2013). 25 latent class analysis and finite mixture modeling. The Oxford handbook of quantitative methods (p. 551).

McClements, L. D. (1977). Equivalence scales for children. Journal of Public Economics, 8(2), 191-210. 
McLaughlin, K. A. (2016). Future directions in childhood adversity and youth psychopathology. Journal of Clinical Child and Adolescent Psychology: the Official Journal for the Society of Clinical Child and Adolescent Psychology, American Psychological Association, Division 53, 45(3), 361-382.

Meng, X., \& D'Arcy, C. (2016). Gender moderates the relationship between childhood abuse and internalizing and substance use disorders later in life: A crosssectional analysis. BMC Psychiatry, 16(1), 401.

Miller, E. K., \& Cohen, J. D. (2001). An integrative theory of prefrontal cortex function. Annual Review of Neuroscience, 24(1), 167-202.

Miller, E., Breslau, J., Chung, W. J., Green, J. G., McLaughlin, K. A., \& Kessler, R. C. (2011). Adverse childhood experiences and risk of physical violence in adolescent dating relationships. Journal of Epidemiology and Community Health, 65(11), 1006-1013.

Muthén, L., \& Muthén, B. (2018). Mplus. The comprehensive modelling program for applied researchers: User's guide (p. 5).

Nantel-Vivier, A., Pihl, R. O., Côté, S., \& Tremblay, R. E. (2014). Developmental association of prosocial behaviour with aggression, anxiety and depression from infancy to preadolescence. Journal of Child Psychology and Psychiatry, 55(10), 1135-1144.

O'Connor, M., Slopen, N., Becares, L., Burgner, D., Williams, D. R., \& Priest, N. (2020). Inequalities in the Distribution of Childhood Adversity From Birth to 11 Years. Academic Pediatrics, 20(5), 609-618. https://doi.org/10.1016/j.acap.2019.12.004

Organization, W. H. (2016). INSPIRE: Seven strategies for ending violence against children. World Health Organization.

Rasmussen, L. J. H., Moffitt, T. E., Eugen-Olsen, J., Belsky, D. W., Danese, A., Harrington, H., \& Williams, B. (2019). Cumulative childhood risk is associated with a new measure of chronic inflammation in adulthood. Journal of Child Psychology and Psychiatry, 60(2), 199-208.

Robins, R. W., John, O. P., Caspi, A., Moffitt, T. E., \& Stouthamer-Loeber, M. (1996). Resilient, overcontrolled, and undercontrolled boys: Three replicable personality types. Journal of Personality and Social Psychology, 70(1), 157-171. https://doi.org/10.1037/0022-3514.70.1.157

Rust, J., Bennun, I., Crowe, M., \& Golombok, S. (1986). The Golombok Rust inventory of marital state (GRIMS). Sexual and Marital Therapy, 1(1), 55-60.

Schneider, R. J., Ackerman, P. L., \& Kanfer, R. (1996). To "act wisely in human relations:" exploring the dimensions of social competence. Personality and Individual Differences, 21(4), 469-481.

Scully, C., McLaughlin, J., \& Fitzgerald, A. (2020). The relationship between adverse childhood experiences, family functioning, and mental health problems among children and adolescents: a systematic review. Journal of Family Therapy, 42(2), 291-316.

Shepherd, P. (2012). Millennium cohort study: Ethical review and consent. Retrieved from https://cls.ucl.ac.uk/wp-content/uploads/2017/07/MCS-Ethical-Approvaland-Consent-2019.pdf.

Silberg, J. L., Maes, H., \& Eaves, L. J. (2010). Genetic and environmental influences on the transmission of parental depression to children's depression and conduct disturbance: An extended Children of Twins study. Journal of Child Psychology and Psychiatry, 51(6), $734-744$.

Slopen, N., Loucks, E. B., Appleton, A. A., Kawachi, I., Kubzansky, L. D., Non, A. L., ... Gilman, S. E. (2015). Early origins of inflammation: An examination of prenatal and childhood social adversity in a prospective cohort study. Psychoneuroendocrinology, 51, 403-413.

StataCorp, L. (2017). Stata statistical software: Release 15 (2017). College Station, TX: StataCorp LP.

Straus, M. A. (1979). Measuring intrafamily conflict and violence: The conflict tactics (CT) scales. Journal of Marriage and the Family, 75-88.

Straus, M. A., Hamby, S. L., Finkelhor, D., Moore, D. W., \& Runyan, D. (1998). Identification of child maltreatment with the Parent-Child Conflict Tactics Scales: Development and psychometric data for a national sample of American parents. Child Abuse \& Neglect, 22(4), 249-270. https://doi.org/10.1016/s0145-2134(97) 00174-9

Tomoda, A., Suzuki, H., Rabi, K., Sheu, Y.-S., Polcari, A., \& Teicher, M. H. (2009). Reduced prefrontal cortical gray matter volume in young adults exposed to harsh corporal punishment. Neuroimage, 47, T66-T71.

Williamson, R. A., Donohue, M. R., \& Tully, E. C. (2013). Learning how to help others: Two-year-olds' social learning of a prosocial act. Journal of Experimental Child Psychology, 114(4), 543-550. https://doi.org/10.1016/j.jecp.2012.11.004

Witt, A., Münzer, A., Ganser, H. G., Fegert, J. M., Goldbeck, L., \& Plener, P. L. (2016). Experience by children and adolescents of more than one type of maltreatment: Association of different classes of maltreatment profiles with clinical outcome variables. Child Abuse \& Neglect, 57, 1-11.

Zolotor, A. J., \& Puzia, M. E. (2010). Bans against corporal punishment: A systematic review of the laws, changes in attitudes and behaviours. Child Abuse Review, 19 (4), 229-247. 